ACTA UNIVERSITATIS LODZIENSIS

Folia Litteraria Romanica 14, 2019

http://dx.doi.org/10.18778/1505-9065.14.06

Sabine Bastian

Universität Leipzig

(iD https://orcid.org/0000-0001-5768-355X

sbastian@uni-leipzig.de

\title{
Christian Oertl
}

Universität Leipzig

(iD https://orcid.org/0000-0001-6782-2226

christian.oert1@googlemail.com

\section{L'alcool et comment on en parle entre jeunes en Allemagne et en France}

\begin{abstract}
RÉSUMÉ
Cet article prend pour sujet des expressions allemandes et françaises dans le domaine de boissons alcoolisées. Comme 11\% des jeunes âgés de 17 ans déclarent boire régulièrement de l'alcool, une recherché comparative dans ce domaine promet une évaluation copieuse vu que ces habitudes linguistiques influencent également la façon dont s'expriment les plus âgés. La plupart du corpus a été récoltée, analysée et discutée lors d'une étude sur le terrain pendant trois semaines dans un des bars les plus populaires parmi les jeunes Leipzigois. Les abréviations constituent le phénomène le plus fréquent, suivies des métaphores ainsi que des expressions ironiques voire satiriques. Des néologismes sont également entrés dans le registre, par exemple l'acronyme Uwe (unten wird's eklig - en bas, c'est dégoûtant), ce qui est une allusion au prénom masculin allemand Uwe et désigne la dernière gorgée d'une boisson. La traduction se présente parfois comme problématique en raison de jeux de mots ou de realia. Cependant, plein d'options lexicales sont disponibles afin de transmettre le message souhaité.
\end{abstract}

MOTS-CLÉS - parler jeune, argot des bars, loisirs, boissons alcoolisées, alcool

\section{Youth Slang Expressions Relating to the Consumption of Alcoholic Drinks in German and French}

\section{SUMMARY}

This article focuses on German and French youth slang expressions that refer to the consumption of alcoholic drinks. Since $11 \%$ of 17 -year-olds admit to consuming alcohol regularly at different places - such as friends' houses, bars, or even school - a cataloguing study of conversational habits in this field promises a productive evaluation as these habits also influence older people's conversational 
habits. The largest part of the corpus has been collected, analysed, and discussed in a three-week field study in one of Leipzig's most popular spots among young people. Short forms are one of the most common expressions used by young people, while metaphors as well as ironic and satiric expressions are just as common. Even new creations have been registered, such as the acronym 'Uwe' ('unten wird's eklig', i.e. 'at the bottom it's nasty'), which is also an allusion to the German male name 'Uwe', but here it refers to the last sip of a beer or any other drink. Translation may seem hard due to puns and a given contextual reality. However, there are many lexical options available to make sure that the intended message is kept alive.

KEYWORDS - youth slang, bar slang, leisure habits, drinks, alcohol

\section{Introduction}

Parlant de sa génération, l'Allemand Amadeus Ulrich (20 ans) cite les dénominations " Generation doof 》 (" génération idiote 》), " Generation sorglos » ("génération sans-souci »), " Generation Internet » (" génération Internet ») et "Generation dick " (" génération grosse »), mais en réalité, cette génération est surtout une "Generation Alkohol » (" génération alcool »). Selon lui, pour un week-end réussi, il faut boire de l'alcool sans retenue dans des beuveries, partouzes " à forfait », débouchant parfois sur une intoxication alcoolique (cf. Ulrich : Zeit online, 15.11.2011).

Les jeunes en France connaissent très bien cette situation qu'ils décrivent comme " fête » qui est une " chouille », forcément alcoolisée. Ils empruntent également des termes anglais comme le " binge drinking $»^{1}$ qu'ils traduisent par « défonce totale» ou « biture express », lorsque les jeunes en Allemagne emploient le terme « Komasaufen », qui signifie « boire jusqu'à l'état comateux ».

C'est le point de départ de notre recherche comparative dans le domaine de la relation entre les jeunes et l'alcool et la façon dont ils en parlent. Il s'agit de faire d'abord un bref inventaire des expressions non-standard actuelles autour des boissons alcooliques et l'acte de boire, notamment auprès des jeunes, mais aussi auprès des plus âgés influencé par le langage des jeunes. Les corpus dépouillés sont tirés d'une part d'Internet, centrés sur les témoignages des adolescents ${ }^{2}$ et d'autre part sur le recueil d'exemples sur le vif dans un bar fréquenté par des jeunes Leipzigois.

En étudiant la sémantique de ces expressions dans leurs contextes et en comparant l'usage des termes français et allemands nous nous posons la question de l'internationalisation non seulement du phénomène de la consommation d'alcool mais aussi de sa mise en mots dans nos deux pays, la France et l'Allemagne. Les exemples choisis ainsi que quelques conseils aux (futurs) traducteurs et

\footnotetext{
${ }^{1}$ Binge-Dringing (2011) : http://jeunes.alcool-info-service.fr/alcool/binge-drinking\#.

2 Melissa (2017) : J'étais alcoolique à 25 ans, voici où j'en suis, deux ans plus tard. Voir également Beaugé et al. 2011.
} 
interprètes ont pour but de démontrer des stratégies linguo-traductologiques. La recherche d'équivalents acceptés par les (jeunes) lecteurs de textes, de romans et de commentaires dans les réseaux sociaux ainsi que les spectateurs de films dans ce domaine pose souvent des problèmes de traduction car les dictionnaires n'indiquent que peu de propositions. Cette quasi-absence d'études germanofrançaises dans le domaine du parler jeune sur la consommation d'alcool cimente notre motivation de débloquer la situation.

La recherche sociologique ayant recours aux statistiques de la consommation des jeunes adolescents indique qu'à 17 ans, l'alcool est la substance psychoactive la plus consommée 3 . Pas moins de $11 \%$ des jeunes âgés de 17 ans déclarent boire régulièrement de l'alcool. À la différence des adultes, la consommation se pratique plutôt le week-end entre amis et non pendant la semaine ou en solitaire. Les lieux de consommation préférés sont les domiciles (privés, des amis ou des parents) et les débits de boissons (bars, pubs, discothèques), suivis des lieux publics ouverts et l'école. Deux tendances à propos de la consommation régulière sont présentes : dans des milieux socio-économiques plus élevés, plus de personnes boivent de l'alcool et, vu leurs ressources financières, ils en ont plus souvent la possibilité que les personnes d'autres milieux. Enfin, la consommation régulière d'alcool est croissante et plus importante dans les familles où les deux parents ne vivent plus ensemble ou dans lesquelles les enfants vivent séparément des parents, en internats par exemple.

Les exemples français et une partie des exemples allemands ${ }^{4}$ ont été repérés chez ceux parlant du problème de la consommation d'alcool. Pour le français nous renvoyons à la recherche de Beaugé et al. qui ont publié sur le site de Lesinrocks. com des listes contenant des termes « jeunes» avec les explications nécessaires. Pour donner quelques exemples, nous citons les suivants :

- Rebié : Verlan du mot bière, la "rebié" a largement supplanté la "binouze", désormais exclusivement consommée par des cadres trentenaires ayant fait tomber la cravate pour la soirée. Ex. : "C'est pas de la rebiè, la Tourtel.

- Rabat : Soûl en langage jeune et urbain. Ex. : "Quatre pattes j'connais ap', même complètement rabat", ainsi que ledit Booba dans son tube Jour de paye.

- Ginto ou vodkato : Car il est parfois trop long de dire gin tonic ou vodka tonic, ou tout simplement parce qu'on n'y arrive plus, la vie a inventé ces incroyables raccourcis-comptoir que sont ginto et vodkato - et que les barmans ont déjà intégrés, rassurez-vous. Ex. : "Un ginto et une vodkato, steuplaît."

L'analyse de ces sites $w^{-5} b^{5}$ a alimenté un corpus qui nous a servi comme référence - ou bien en termes traductologiques - comme outil nécessaire à trouver des équivalents potentiels tirés de textes parallèles.

\footnotetext{
${ }^{3}$ Bertrand 2018.

${ }^{4}$ Ulrich 2011.

5 Nous renvoyons pour d'autres exemples aux sites Nouvelobs : « Beuveries express » (2013), Mahuzier 2013, Prémix (2015) sur filsantéjeunes.com ou textfocus.net.
} 
Dans les sections suivantes, nous nous penchons sur la partie centrale de notre recherche actuelle qui porte sur les exemples de l'allemand qui ont été relevés sur le terrain, dans un bar.

\section{Lieu et méthode de la recherche}

Le bar s'appelle Jet et se trouve depuis six ans dans un quartier étudiant de Leipzig. Le concept de ce bar est d'offrir au public un salon ou plutôt une salle de jeux. Sur environ $400 \mathrm{~m}^{2}$, le lieu offre des baby-foots, des jeux vidéo, des jeux de table, de ping-pong etc. Il n'existe pas de service sur table, les boissons sont vendues au bar ce qui en fait en quelque sorte le centre social de l'établissement. Le public est très varié, mais le noyau dur est constitué par des jeunes entre 20 et 30 ans, ayant souvent le statut d'étudiant dont la majorité habite ou semble habiter à Leipzig ou dans les alentours. Cependant, comme la ville attire des gens de toute l'Allemagne, un grand nombre d'entre eux vient d'autres régions (en plus des touristes). La grande offre de différentes boissons, surtout alcoolisées et provenant du pays et même du monde entier, promet un vaste répertoire de désignations alternatives. La variété du corpus établi correspond à celle du public et de l'offre même.

La méthode choisie était assez simple : servir les clients et prendre en notes les expressions repérées dans les dialogues. Toutes les entrées ont été cataloguées et classées. Au fur et à mesure, le corpus a été présenté et partagé avec le public. Les discussions issues de ces présentations ont contribué à mieux comprendre et définir les expressions fréquentes. Celles-ci sont le vrai objectif de cette recherche : les mots et expressions connus à travers le public et non celles qui sont trop individuelles. En discutant les entrées, elles obtiennent leur légitimation pour ainsi dire. C'est l'usage et en même temps le savoir commun de ces expressions familières qui en font des expressions établies. Le public est plutôt jeune et provient surtout du milieu universitaire, l'enquête est empirique et non exhaustive et ne couvre pas toutes les classes d'âge ou couches sociales; elle ne donne qu'un aperçu d'un certain public dans un certain endroit dans un certain espace de temps.

\section{Quelques résultats}

\section{1. Économie par abréviations et minimisations}

Qui l'eût cru ! Les abréviations classiques constituent les formes les plus répandues et servent un aspect central du langage familier : l'économie linguistique, ce qui est parfois nécessaire dans un bar, notamment dans le rush. Certaines entrées se qualifient en plus d'être interprétées en tant que minimisations de la boisson, 
souvent d'une bière. Ces minimisations paraissent en allemand souvent en forme de «- $i »$, «-lein » ou de «-chen » qui remplacent la dernière syllabe du mot en question. L'omission de la dernière syllabe, d'une autre syllabe ou d'une part d'un mot composé paraît aussi fréquente.

- Sternburg Export :

Export, SteBu, Sternilein, Sterni

- Wicküler :

Wicki, Wickilein

- Ur-Krostitzer :

Krosti, Urilein, Uri

- Pilsner Urquell :

$P U$

- Budweiser :

Bud

- Augustiner :

- Schöfferhofer Grape Fruit :

Augustinerchen, Gustl

- Schöfferhofer Weizen :

- Staropramen :

Grape

Schöffchen, Schöff, Schöffi

- Jägermeister :

Staro

- Pfefferminzlikör :

Jägi

- Berliner Luft :

Pfeffi

Les exemples ci-dessus sont tous inspirés de noms de marques. La bière Augustiner montre une spécificité : comme cette marque de bière provient de Bavière, la lettre « $l$ » est ajoutée à l'abréviation comme il est d'usage dans cette région d'Allemagne. Le même principe d'abréviations et de minimisations peut être constaté pour les noms propres d'autres boissons.

- Cuba Libre :

Cuba

- Wodka-Energy :

Wodka-E

- Wodka-Orangensaft :

Wodka-O (jus d'oranges)

- Moscow Mule :

Moscow, Mule

- Blaue Lagune :

- Caipirinha :

Lagune (lagune bleue)

Ces expressions sont typiques du lexique quotidien d'un bar. La méthode cependant est appliquée par tout le monde afin de créer des minimisations en allemand :

- Bier (bière) - Bierchen, Kaffee (café) - Käffchen, Spiel (jeu) - Spielchen, Pullover (pull) - Pulli, Spiegel (miroir) - Spieglein etc.

\subsection{Allusions et désignations ludiques dans les produits}

À partir des noms de marques et des noms propres des boissons, la fonction ludique du langage du public fonctionne pleinement. Soit pour se divertir, soit pour se moquer - la créativité ne connaît pas de limites et enrichit l'ambiance. De telles expressions montrent un haut degré de connotations qui parfois nécessitent une explication, ceci au risque de ne pas comprendre, surtout à la première écoute. Ces expressions sont pourtant bien établies, car souvent il suffit d'une seule écoute 
pour se souvenir du message. Parfois, les expressions sont caractérisées par des détails régionaux ou locaux, relatifs à l'origine du produit.

- Jever Fun :

Jever No Fun

Ici il s'agit d'une bière allemande sans alcool, Jever Fun, ce qui initie les gens à des commandes disant No Fun. Le mot anglais fun promet du plaisir, mais donné que cette bière ne contient pas d'alcool, où est le fun ? C'est ce que pensent probablement les clients en la désignant de manière ironique ou même satirique. Voici un autre exemple de la créativité :

\section{- Beck's : \\ Fischpisse (pisse de poisson)}

Sans connaitre le contexte pas de chance de comprendre comment cette expression s'est manifestée. D'abord, le siège de la brasserie Beck's se trouve à Brême dans le nord de l'Allemagne. Un préjugé dit que les gens dans le nord de l'Allemagne ont une affection pour le poisson et la pêche, d'où l'explication pour la partie Fisch (poisson). L'autre partie, -pisse (pisse), désigne la forme liquide $\mathrm{du}$ produit, certes d'une manière très rude. Mais cette rudesse est au centre du message désiré, à savoir que l'on n'aime pas cette boisson ; voilà pourquoi on en parle tellement mal. Une deuxième explication ${ }^{6} \mathrm{~s}^{\prime}$ appuie sur un dicton connu dans le monde du foot national :

- Was ist grün und stinkt nach Fisch? Werder Bremen! (Qu'est-ce qui est vert et pue le poisson? Werder Brême!)

Ce dicton implique la même allusion en ce qui concerne le siège de Werder Brême, un club de foot, et la marque de bière. Comme Brême est connu pour les deux, ces allusions tendent à se répéter en vue d'autres produits issus de Brême. Dans des cas plus généraux, le public emploie le mot «Plörre » qui désigne à l'origine un café très léger, voire trop léger, insipide, pour être savouré.

L'origine d'un produit est très apte à être inclue :

\section{- Tyskie \\ Pilski}

Cette bière polonaise, une bière blonde et donc une pils, incite la créativité à cause de son origine en Pologne. Comme la syllabe «ski» semble typiquement polonaise aux Allemands, ils utilisent «ski» afin de donner un air polonais à n'importe quoi, mais surtout pour s'amuser. Une Pilski signifie alors une bière blonde polonaise, ou dans le cas du corpus et du bar, la marque Tyskie qui y est vendue.

Parfois le nom propre de la boisson fait partie d'une expression ou d'une intervention entière :

- «Einen Cuba Libre bitte, aber muy libre! » («Un Cuba Libre s’il vous plait, mais muy libre! »)

Le client demande alors un cocktail Cuba Libre avec plus d'alcool que d'usage en se servant de l'adverbe espagnol « muy » en combinaison avec l'adjectif « libre » et joue ainsi avec la langue afin d'obtenir sa boisson « muy libre », « très libre » alors. ${ }^{6}$ Fischpisse est à rapprocher du français pisse de chat, qui désigne une bière légère en alcool sans
véritable goût. 
La même tendance ludique est constatée de manière encore plus importante pour des expressions plus générales, sans noms propres ou noms de marques. Le langage devient ironique, faisant allusion de manière métaphorique en grande partie, comme le montre l'exemple suivant du terme " Fassbier » (" bière à la pression $\gg)$ :

- Fassbier

- Fass : abréviation classique

- Fassbrause : expression ironique car la Fassbrause est une limonade sans alcool, mais qui est souvent servie à la pression

- Eins vom Hahn : abréviation de "Zapfhahn » (« robinet »), "une du robinet »

- Gezapftes : " tiré au tonneau », basé sur l'activité

- Rohrperle : "la perle du tuyau », expression ironique car la bière vient d'un tuyau, aussi utilisé pour l'eau du robinet et donc dans le double sens probablement ironique

- Leitungswasser mit Schuss : " eau du robinet avec une gorgée d'alcool », expression ironique visant au fait que la bière vient du tuyau et contient de l'alcool.

La même tendance est à observer pour le terme "Biermischgetränk" ( "boisson mélangée à base de bière ») :

- Biermischgetränk

- Radler / Alster : expressions standardisées pour une bière mélangée avec de la limonade. "Radler » signifie " cycliste », alors quelqu'un qui ne doit pas boire trop de bière afin de rester en état de conduire, pendant que "Alster » est le nom d'un fleuve

- Diesel : expression plus ou moins standardisée pour une bière mélangée avec du coca. "Diesel » (" gazole ») semble déduit de la couleur du carburant Diesel

- Saures : " aigre », expression récente pour une bière mélangée avec de l'eau minérale

- (Bier-) Limonade : "limonade (à la bière) », expression ironique car la bière est mélangée avec une limonade et donc moins forte, comme une limonade

- Mädchenbier : " bière pour filles 》, allusion au stéréotype que les femmes boivent surtout des bières moins fortes ou mélangées et que les hommes sont plus aptes à boire de la vraie bière

- Bierschorle : le mot "Schorle » signifie une boisson mélangée à base d'eau gazeuse et d'une autre boisson (vin, jus de fruits...) et donc "Bierschorle » est en général le mélange de bière et d'une autre boisson.

La boisson non-alcoolique dans ces mélanges, le filler dans le jargon des bars, est dénommée "Mixgelumpe », ce qui donne une impression plutôt péjorative à cause de l'allusion aux « loques » soit pour les bières, soit pour d'autres boissons, 
même s'il s'agit d'un produit de haute qualité comme le coca. La forme des boissons, plus précisément la forme de la bouteille, n'attire que peu d'attention lors la création de nouvelles expressions. Les notes prises ont enregistré quelques exemples quand même pour décrire " Flaschenbier " (" bière en bouteille »), «Kanne » («pot»), « Hülse » («pellicule »), «Pulle» («boutanche »).

Les eaux-de-vie, elles aussi, n'attirent que peu l'attention non plus en ce qui concerne des expressions alternatives à « Schnaps », "Kurzer» (petit verre de schnaps) ou "Shot » (emprunté de l'anglais). Seul le mot «Rachenputzer» ( "piquette», litt. nettoyeur de gosier) montre une certaine créativité et a été utilisé exclusivement en combinaison avec des alcools plus forts. Afin de dénommer le contenu, un spiritueux, le mot «Sprit » («carburant») est employé car il fait rouler soit la voiture, soit le consommateur. À propos du consommateur : plein d'expressions sont dédicacées à toute personne qui en boit trop de temps en temps ou régulièrement. Voici quelques exemples :

- Spritti : dérivédu mot «Sprit» («carburant») ou «Spirituose 》 (« spiritueux »), désigne quelqu'un qui boit souvent de grandes quantités d'alcools forts

- Alki : abréviation du mot "Alkoholiker», désigne un alcoolique, un alcoolo

- Besoffski : mélange du mot «besoffen » (" soûl ») et de la syllabe polonaise "-ski », allusion au cliché que les Polonais boivent souvent et assez d'alcool

- Trunkenbold : soûlard.

En Allemagne un sondage de type humoristique remontant à quelques années comportait la question : comment appeler la dernière gorgée d'une bouteille ou d'un verre ? La réponse la plus fréquente était «Uwe », ce que l'on constate également dans notre enquête. Uwe, un prénom allemand traditionnel, est aussi l'acronyme de l'énoncé « unten wird's eklig 》 (" en bas, c'est dégoûtant 》), raison pour laquelle ce mot simple s'est largement propagé. D'autres variantes sont d'une part «Pennerschluck» (" gorgée des clochards »), lorsque les consommateurs sont d'accord sur le fait qu'il ne faut pas boire ce reste, d'autre part « Spuckschluck» ("gorgée à la salive ») du fait qu'à chaque gorgée on perd de la salive dans la bouteille jusqu'à ce qui ne reste que de la salive.

\subsection{Aller boire un coup... ou ?}

Comment parler du sujet d'aller boire un coup sans recours à la langue standard ?

La situation au départ de chaque enquête sur les parlers dans un bar est la commande des boissons. L'expression « ein kühles Blondes" (« une bière blonde fraîche ») se trouve régulièrement parmi les interventions et peut désigner - en théorie - chaque bière blonde. Le contenu informatif d'une telle intervention est donc peu élevé car il est peu explicite. L'énoncé bref «noch eins, bitte» (« encore une, s'il vous plait ») par contre est également très répandu et beaucoup plus précis car dans une telle conversation, on voit directement ce que le client demande. De 
plus, on n'utilise pas plus de langage que nécessaire pour exprimer le message. Dans un tel cas, le langage utilisé est alors marqué par un haut degré d'économie linguistique. Cela s'applique aussi aux expressions «nachladen » ("recharger»), " aufladen » (" charger ») ou bien " die Luft rauslassen » ("faire sortir l'air de la bouteille / du verre »).

Le processus initiateur pour boire un coup - «etwas trinken gehen 》- est aussi soumis à de nombreuses créations :

- einen heben : dérive du verbe lever, par exemple lever un bras afin de boire un coup

- einen kippen : dérive du verbe verser

- einen picheln : picoler, boire un petit coup entre amis

- einen bechern : verbe dérivant du nom «Becher » (gobelet)

- einen hinter die Binde kippen : s'enfiler un autre verre dans le gosier

- einen löten : souder

- einen zischen : chuinter, expression très onomatopéique car le bruit rappelle l'eau qui tombe dans une poêle chaude ou le bruit qui se produit en ouvrant une bouteille

- einen ballern : tirer des coups de feu, surtout utilisé en buvant des Schnaps

- einen kloppen : dérive du coup, également surtout utilisé en buvant des Schnaps

- einen reinzwirbeln : tortiller

- eine Ziehung machen : faire un tirage

- eine Session machen : faire une séance

- Saufi-Saufi : nom dérivé du verbe "saufen » (picoler, boire de l'alcool)

- einen reindrücken : pousser

Cette liste n'est pas exhaustive et ne montre que quelques exemples parmi les plus fréquents. Presque toutes ces expressions s'accompagnent du verbe " gehen» (« aller ») afin d'annoncer le plan, par exemple « einen picheln gehen » (« aller en picoler un 》) ou d'autres auxiliaires, par exemple « einen picheln wollen » ( « vouloir en picoler un »).

Après avoir bu trop d'alcool de nombreuses expressions et définitions de cet état se rajoutent au relevé parfois métaphorique. En voici quelques exemples :

- voll wie eine Haubitze sein : être soûl comme un obusier

- einen sitzen haben : être éméché

- sternhagelvoll sein : être raide

- voll wie ein Russe / Pole sein : être soûl comme un Russe / un Polonais

- Oberkante Unterlippe sein : être soûl jusqu'à la partie supérieure de la lèvre inférieure

- den Juhnke machen : faire le Juhnke, allusion à l'acteur allemand Harald Juhnke qui était connu pour son alcoolisme

- einen im Tee haben : avoir un coup dans l'aile

- das letzte Bier / Getränk war schlecht : la dernière bière / boisson était mauvaise. 
Cette dernière entrée signifie clairement une mauvaise excuse pour avoir bu trop d'alcool, mais la solution a déjà été inventée : le «Konterbier» («bière contreattaque ») que les Allemands boivent afin de «den Pegel halten » (" contrôler le fluviomètre »), rester assez alcoolisé alors, et de ne pas souffrir d'une gueule de bois, ou au moins afin de justifier une telle consommation. La boisson alcoolique préférée des Allemands, la bière, est aussi consommée en forme de "Wegbier » ( «bière pour la route »), lorsqu'on est en train d'aller vers un but, par exemple une fête, un concert etc. Cette même idée est désignée par l'expression «Fußpils», une bière blonde qu'on boit en allant à pied, qui en même temps fait allusion au mot «Fußpilz» (« mycose des pieds »). Il existe même une expression pour l'activité de boire une bière en se promenant sans but, par exemple en été dans les parcs : "Spazierbier », la bière pour faire une promenade, fidèle à la devise que le chemin est le but. Ceux qui n'aiment pas la bière trouvent probablement leur bonheur avec la «Mische », un mélange contenant de l'alcool, d'habitude un spiritueux, et un jus de fruits ou un soda. La «Mische », dérivé du mot «Mischung» («mélange»), n'est cependant pas bue dans les bars ou clubs, mais uniquement en route, au bord d'un lac, dans les parcs et tout autre lieu sans impératif de consommation, c'est donc une boisson mélangée par le consommateur même.

Assis sur un banc, muni d'une Mische, d'une Wegbier ou d'une Spazierbier, les activités continuent. Le verbe " cornern » signifie l'acte d'être assis sur un banc public et d'observer les passants, de disserter sur la vie des passants, etc. Un autre jeu autour de la consommation d'alcool est appelé « Spiel des Lebens » ( « jeu de la vie »). Ce jeu consiste à toujours marquer sa bouteille de bière. Si l'on oublie de marquer sa bouteille, un autre joueur pose la question "Wie steht es um dein Spiel ? » («Comment va ton jeu ? ») et gagne une bière aux dépens de celui qui a oublié de marquer sa bouteille. Ce jeu a été au départ populaire dans les internats. Un jeu que presque tout le monde connaît, par contre, porte le nom «Ex oder Arschloch» («Ex ou enculé»). Avec cet acte de langage, il est demandé à chaque personne présente de finir sa boisson d'un coup ( «auf ex»), ceux qui ne réussissent pas étant alors traités d'enculés. Ce jeu est dénommé aussi «Ex oder Jude » («Ex ou Juif»), ce qui marque une allusion au stéréotype que les juifs sont avares et donc ne finissent pas la boisson d'un coup. La locution fonctionne avec n'importe quelle insulte ce qui en fait un des toasts les plus populaires, surtout en buvant des schnaps ou avant de partir chez soi ou ailleurs.

La langue allemande familière crée des néologismes pour dénommer toutes sortes d'activités, d'états etc. En voici encore deux exemples :

- «nasser Hund »: « chien mouillé », désigne une bouteille de bière dans un casier Sternburg qui ne semble pas bonne, soit pour le goût, soit pour le degré de l'ivresse. Clairement un mythe, une légende pour s'amuser

- «Kotzen führt nicht zur Disqualifikation »: « vomir ne mène pas à la disqualification », un dicton que l'on entend surtout lors des jeux à boire ou des tournois de bière-pong, un jeu qui consiste à jeter des ballons et boire de la bière. 
Après quelques tours, quand l'ivresse se fait remarquer et que les premiers joueurs risquent de capituler, on encourage ainsi leurs partenaires à continuer.

Ces exemples ont été relevés dans un laps de temps d'environ trois semaines, ce qui montre qu'il existe un nombre important de termes ou expressions du langage non-standard à recueillir. Une enquête plus large serait utile afin de se faire une meilleure idée de la façon dont parlent les jeunes à propos d'aller boire un coup et de boire en général. Les termes et expressions relevés connaissent souvent une reprise par les plus âgés, au moins les termes et expressions les plus répandus. Le langage des plus vieux est, dans ce domaine, inspiré par les jeunes et perçu comme enrichissement du lexique. Une fois jugées valables, les expressions sont mémorisées et exploitées selon l'humeur des locuteurs, pourvu que l'on se trouve dans une situation appropriée à l'utilisation de telles expressions.

\section{En guise de conclusion : comment traiter les problèmes de traduction qui s'imposent}

La traduction de telles expressions peut causer des difficultés car le traducteur est tenu de veiller aux traditions et normes de la culture source ainsi qu'à celles de la culture cible. En plus, dans le sous-texte, les attitudes des usagers de la langue par rapport aux normes de la langue parlée doivent être englobées dans une traduction potentielle. Comme l'enquête l'a montré, il existe déjà un nombre de traductions officielles ou standardisées de quelques expressions du milieu de la boisson. Mais comment traduire des expressions qui n'ont pas encore été traduites ? C'est simple pour les diminutions ou les minimisations : l'objectif du traducteur est alors une atténuation, une version plutôt euphémique du terme standard ; la création lexicale peut donc utiliser les procédés habituels. Dans les autres cas, la créativité ou bien l'expérience de telles situations dans les deux cultures / langues doit être exploitée, ce qui pose des problèmes vu la diatopie de l'allemand. D'après Freunek, il semble souvent presque impossible de créer dans la version allemande un équivalent aussi intense et fréquent que dans le texte source sans qualité diatopique. La diatopie serait déjà presque inévitable en utilisant la langue allemande standard à cause de son haut degré de variabilité ( $c f$. Freunek, 2007 : 185). Il reste alors la création d'une traduction aussi proche et raisonnable que possible, car ce qui compte, en effet, est la transmission des associations qui s'imposent dans de telles expressions. Soûl comme un Polonais, comme un Russe, comme un obusier - le message reste intact, ce n'est que le répertoire lexical qui change selon les problèmes de traduction qui s'imposent. Santé ! 


\section{Bibliographie}

Beaugé, Marc, Lisarelli, Diane, Siankowski, Pierre (2011) : Petit lexique moderne de la gueule de bois. http://www.Lesinrocks.com, 31/07/11. Consulté le 20.01.2019

Bertrand, Ornella (2018) : Les jeunes \& l'alcool. Sociologie de la consommation des jeunes adolescents. https://www.alcoolassistance.net/les-jeunes-alcool. Consulté le 20.01.2019

Beuverie express (2013) : « Beuverie express » : Le mot français pour " binge-drinking », Nouvel Observateur 28 juillet 2013, http://www.nouvelobs.com/culture. Consulté le 20.01.2019

Binge-Drinking (2011) : http://jeunes.alcool-info-service.fr/alcool/binge-drinking\#. Consulté le 24.01.2019

Freunek, Sigrid (2007) : Literarische Mündlichkeit und Übersetzung : am Beispiel deutscher und russischer Erzähltexte, Berlin, Frank \& Timme, 2007

Mahuzier, Marc (2013) : Les jeunes et l'alcool : comment ils en parlent. In : La Matinale / La monet le 27/09/2013 à $06: 51$, http//www.ouest-france.fr (Journal numérique). Consulté le 24.01.2019

Melissa (2017) : J'étais alcoolique à 25 ans, voici où j'en suis, deux ans plus tard. http://www. madmoizelle.com. Consulté le 24.01.2019

Prémix (2015) : Les prémix et autres boissons " pour jeunes ». http://www.filsantejeunes.com. Consulté le 20.01.2019

Rieder, Caroline (2012) : Les jeunes et l'alcool, un dangereux tandem qui commence à 12 ? ans. http://www.24heures.ch. Consulté le 24.01.2019

Textfocus (2019) : http://www.textfocus.net. Consulté le 24.01.2019

Ulrich, Amadeus (2011) : Generation Alkohol. http://www.zeit.de, 15 novembre 2011 (zeit online). Consulté le 6.06.2018

Sabine Bastian - est professeure émérite des Universités. Elle a enseigné pendant plus de 40 ans à l'université de Leipzig dans la formation des traducteurs et interprètes de français et de l'allemand aussi bien que des futurs professeurs de français. Dans sa recherche sociolinguistique elle privilégie depuis longtemps les parlers jeunes, leur description et les problèmes de leur traduction.

Christian Oertl - a fait ses études de traduction et d'interprétation de conférences à Leipzig et à Bruxelles. Après quelques ans de travail en tant que traducteur, interprète et enseignant, il se dédie dans sa thèse à la science des mèmes et comment ce phénomène d'Internet diffuse des clichés, stéréotypes et préjugés. 